\title{
RETROPERITONEAL TERATOMA IN INFANCY
}

BY

\author{
DAVID CHARLES \\ From the Institute of Obstetrics and Gynaecology, Hammersmith Hospital
}

(RECEIVED FOR PUBLICATION JANUARY 1, 1953)

The first authentic description of a case of retroperitoneal tumour is ascribed to Morgagni in 1761. Since that time only 82 cases of retroperitoneal teratomata have been published in the literature.

These tumours lying preaxially must be regarded as pathological manifestations of a physiological process (Nicholson, 1929). Although all three germinal layers are present in teratomata, there is no segregation of tissues. The old theory that these tumours resembled a twin foetus enclosed in another twin is no longer valid although teratoma and host lead an asymbiotic existence. As they contain all three embryonic layers in no standard pattern they must be present at an early stage of foetal development, and further, as they are extraperitoneal and in the region of the primitive mesentery, retroperitoneal teratomata are in a nidal state before the peritoneum is evolved (Willis, 1935). In the case presented, all somatic tissue elements were present but no regional segregation of tissues was observed and at operation the mesenteric relationship of the tumour was clearly defined.

Spemann (1938) elaborated the theory that primary organizers influenced the embryonic tissues and stated that cells escaping their action are derivatives of or related to the invaginating primitive streak, and thus as teratomata arise in a median or paramedian location they may escape organizer influence. This theory furthers the view now generally accepted that teratomata are not incomplete foetuses but derangements of development arising during the pre-somite stage.

\section{Case Report}

S.G. (No. 13071), a girl aged 2 years 2 months, was admitted to the Gloucester Royal Hospital on June 27, 1949. She had been born prematurely after about 36 weeks' gestation. The mother had toxaemia of pregnancy and labour was completed by a forceps delivery. The infant had a left-sided facial paresis which cleared up satisfactorily. Birth weight was $5 \mathrm{lb}$. $12 \mathrm{oz}$. The mother had had one uneventful pregnancy seven years previously.

The child developed quite normally and at no time was there concern about her progress.

She was very well until three days before admission to hospital. when her appetite was poor and she complained of pains in the region of the umbilicus. There had been no vomiting or urinary disturbance.

The child's father had a past history of phthisis and five years previously the patient's brother had died of tuberculous peritonitis.

On examination she was seen to be a well built child (weight, $30 \mathrm{lb}$., and height, 28 inches). The face was highly coloured, and the skin was moist but not hot. There was no cyanosis or icterus. The temperature was $100 \cdot 4^{2}$, pulse 120 , and respirations 22 . There were no abnormalities of the eyes, ears, nose or throat. The cardiovascular system, respiratory and central nervous systems were normal.

A large, firm, ovoid mass situated to the right of the mid-abdominal line, extending approximately from 2 inches above the umbilicus to 3 inches below, was easily palpable. It was not fixed to the anterior abdominal wall, but seemed to be attached to the coils of overlying gut. The mass had a definite upper border and was separate from the liver. It did not move with respiration and was mobile in the oblique axis from the right iliac fossa to the left hypochondrium. It could not be displaced into the right loin and no pain or tenderness was elicited on palpation. On rectal examination, the mass could be felt on the right side. There was no other clinical abnormality.

Investigations, The urine contained a trace of albumin, but no cells or casts were seen.

Blood was Group A Rh positive, and a full count gave haemoglobin $85 \%$ (Haldane), red blood cells 4,750,000 per c.mm., leucocytes 12,400 (polymorphs $39 \%$, lymphocytes $52 \%$, monocytes $4 \%$, eosinophils $4 \%$, basophils $1 \%)$. The Wassermann reaction was negative.

A Mantoux test $(1 / 10,000)$ was negative.

Radiographs of the chest showed no abnormality. Radiographs of the abdomen showed that the right psoas shadow was obscured. The ascending colon, which was outlined by gas, was displaced upwards. On the medial side of the ascending colon a tooth and a small calcified area were seen.

An intravenous pyelogram showed no abnormality.

Operation. Premedication was with 'nembutal', grain 1 , and atropine, grain $\frac{1}{100}$. The anaesthetic was nitrous oxide, oxygen and ether.

The abdomen was explored through a right paramedian incision. There was no ascites and the kidneys, liver, gall bladder, spleen and pelvic organs showed no abnormality. An oval tumour lay retroperitoneally in the root of the 
mesentery of the small gut. The surface of the mass was lobulated but no cystic areas were noted.

The post-parietal peritoneum was incised lateral to the ascending colon and the colon reflected medially. The tumour was then mobilized and removed. The gut showed no evidence of any impairment to its blood supply on completing the operation and the abdominal wound was closed in layers.

At the end of the operation the child's condition showed no cause for concern and convalescence was uneventful. She was discharged from hospital on the twenty-second post-operative day. The child has since been seen at frequent intervals. Clinically and radiologically there is no evidence of recurrence and she is in good health.

Pathology. The specimen was a cystic tumour weighing

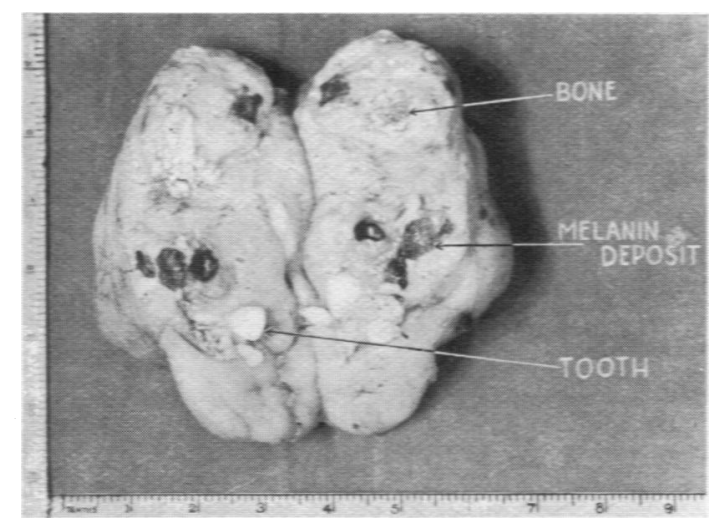

Fig. 1. Cross section of teratoma illustrating melanotic deposits and teeth.

17 ounces. It was ovoid, measuring about 5 in. in its long axis. The surface was irregular on the ventral aspect but smooth on the dorsal surface. The tumour was partially covered by fibrous adhesions. On section, the teratoid nature of the tumour was readily observed. Dental, osseous, myxomatous and epidermal tissues were noted.

Tissues derived from all three embryonic layersectoderm, mesoderm and endoderm-could be seen microscopically in different parts of the tumour. There was much brown pigmentation (probably melanin) in some areas.

\section{Discussion}

In this case, on account of a family history of tuberculous infection the differential diagnosis had to include encysted plastic peritonitis. As the child had a raised temperature this possibility had to be seriously considered. The Mantoux reaction, which was negative using $1 / 10,000$ old tuberculin, did not rule out tuberculous infection but the physical findings together with the Mantoux test made that diagnosis improbable.
A diagnosis of a Wilms tumour was also entertained, but as the mass could not be displaced into the right loin and intravenous pyelography showed no abnormality of the renal tract, this was not considered further.

Arnheim (1943) states that a normal pyelogram does not rule out embryoma of the kidney from the differential diagnosis of an abdominal tumour, but in the literature on Wilms's tumours radiological findings usually settle the diagnosis.

In the present case pressure symptoms due to the tumour were completely absent. The symptoms described in other cases include backache, oedema of the legs and distended veins on the abdominal wall. Again referring to the low grade fever on admission to hospital, which was of unknown origin, Mecray and Frazier (1937) state that it occurs with retroperitoneal tumours but usually indicates the presence of a sarcoma or sarcomatous change. Neither sarcomatous change nor other condition was present in this case so the teratoma must have caused the raised temperature.

The possibility of the mass being a neuroblastoma was considered, but in this condition there is often a history of loss of weight associated with fatigue. Neuroblastomata are usually medially situated and more fixed and nodular in character than teratomata. The radiological finding of a tooth is the main method of differentiating these tumours before operation in the early stages; naturally in the later stages of neuroblastomata secondary involvement of other organs will help in reaching a diagnosis.

Mesenteric cysts also enter into the differential diagnosis and here again radiology is of great assistance as only the large cysts are fluctuant on clinical examination.

Retroperitoneal teratomata are not malignant, but one of their component structures may undergo malignant change at a later date if operative removal at an early stage is not carried out.

\section{Summary}

A case of a retroperitoneal teratoma in a child is described.

I wish to thank Mr. P. M. Birks, under whose charge the child was admitted, for permission to publish this case report.

\section{REFERENCES} Arnheim. E. E. (1943). J. Mt. Sinai Hosp., 10. 355.
Mecray, P. M. and Frazier, W. D. (1937). Arch. Surg., Chicago, 35. 358

Nicholson. G. W. de P. (1929). J. Path. Bact., 32, 365

Spemann, H. (1938). Embryonic Development and Induction. Newhaven. Connect.

Willis, R. A. (1935). J. Path. Bact., 40. 1. 